\title{
OTIMIZAÇÃO DO PROCESSO NÃO INVASIVO E NÃO DESTRUTIVO DE EXTRAÇÃO E ANÁLISE DE METABÓLITOS VOLÁTEIS DE MAMÃO PAPAIA POR SPME-GC-MS
}

\author{
Larissa Rocha Terra ${ }^{\mathrm{a}, \mathrm{b}}$, Marcia Miguel Castro Ferreira ${ }^{\mathrm{a}}$, Daniel Terao ${ }^{\mathrm{b}}$ e Sonia Claudia do Nascimento de Queiroz ${ }^{\mathrm{b}, *, \mathbb{0}}$ \\ anstituto de Química, Universidade Estadual de Campinas, 13083-970 Campinas - SP, Brasil \\ ${ }^{b}$ Embrapa Meio Ambiente, 13083-970, Jaguariúna - SP, Brasil
}

Recebido em 11/05/2020; aceito em 29/06/2020; publicado na web em 10/08/2020

\begin{abstract}
OPTIMIZATION OF THE NON-INVASIVE AND NON-DESTRUCTIVE EXTRACTION PROCESS AND ANALYSIS OF PAPAYA VOLATILE METABOLITES USING SPME-GC-MS. The analysis of volatiles is an alternative in the routine of quality control of fruits, since the volatile metabolites can change in the presence of microorganisms. A well-established method for extracting volatiles is the solid phase microextraction (SPME). This work aims to define some parameters (fiber coating, conditioning time and fiber exposure time) for an effective extraction. Whole papaya was inserted into a hermetically closed glass bottle and the system was left in conditioning for the volatiles accumulation. Then, extraction by SPME and subsequent desorption in a gas chromatograph was performed. Two fibers were evaluated: a $100 \mu \mathrm{m}$ polydimethylsiloxane (PDMS) and a $65 \mu \mathrm{m}$ polydimethylsiloxane/divinylbenzene (PDMS/DVB). The PDMS/DVB fiber extracted the highest number of volatiles. Central composite design (CCD) was performed to assess the effect of papaya conditioning time and the fiber exposure time on the response: number of detectable peaks. Both variables studied and their interactions were significant for the response. ANOVA showed that the quadratic terms and the lack of fit were not significant $(\mathrm{p}<0.05)$. A conditioning time of $10 \mathrm{~min}$ and exposure time of $30 \mathrm{~min}$ was sufficient for the detection of more than 100 compounds.
\end{abstract}

Keywords: central composite design; response surface methodology; metabolomics; fruit.

\section{INTRODUÇÃO}

O mamoeiro (Carica papaya L.) é uma planta dicotiledônea pertencente à família Caricaceae. Ele tem ciclo semi-perene, com pico de produção entre 3 e 5 anos, sendo, portanto, uma cultura de grande extensão agrícola. ${ }^{1}$ Essa espécie é original da parte tropical das Américas, e seu fruto (mamão papaia), desde muitos anos, é amplamente consumido devido ao sabor agradável e ao seu alto valor nutricional e medicinal, já que possui elevado teor de vitaminas A e C, minerais, carotenoides e açúcares. ${ }^{2}$ Devido às propriedades laxantes $\mathrm{e}$ desintoxicantes, o consumo de mamão maduro ou seu suco melhoram o funcionamento do sistema digestivo e ajudam a depurar as toxinas e resíduos que se acumulam no intestino, aliviando as infecções do cólon e melhorando o seu funcionamento de modo geral. Além disso, existem relatos da eficácia do suco no tratamento de verrugas, câncer, tumores e induração da pele. ${ }^{2}$

O Brasil destaca-se por ser um dos maiores produtores de mamão do mundo, ficando atrás apenas da Índia, ${ }^{3}$ e a exportação tem sido crescente nos últimos anos, sendo que em 2015 houve um incremento significativo de $18,2 \%$ na exportação da fruta. ${ }^{4}$ Concomitantes à rápida expansão da fruticultura brasileira cresceram os problemas fitossanitários e, dentre eles, as doenças causadas por fungos que geram grandes perdas em pós-colheita. Segundo a FAO (do inglês, Food and Agriculture Organization of the United Nations), estima-se que um terço das frutas e olerícolas se perdem depois de colhidas. ${ }^{5}$

$\mathrm{O}$ aroma e o sabor estão diretamente ligados à percepção da qualidade das frutas. Tais características sensoriais envolvem, na maioria dos casos, compostos orgânicos voláteis (VOCs- do inglês volatile organic coumpounds) tais como, benzilisotiocianato, terpenos, hidrocarbonetos, ésteres, aldeídos, cetonas, álcoois e ácidos orgânicos. ${ }^{6}$ A composição de tais metabólitos voláteis pode ser alterada pela presença de micro-organismos, que causam uma modificação no

*e-mail: sonia.queiroz@embrapa.br metabolismo da fruta, resultando na síntese de novas substâncias ou na mudança dos níveis das já existentes. ${ }^{7}$ Dessa forma, a análise dos VOCs é uma possível alternativa na rotina de controle de qualidade de frutas para a detecção precoce de micro-organismos. ${ }^{8}$

O estudo da fração de voláteis de alimentos consiste em três passos fundamentais: extração, separação cromatográfica e identificação dos compostos. ${ }^{9}$ Um método bem estabelecido de extração de voláteis é a microextração em fase sólida (SPME), que é uma técnica de preparação de amostras simples e eficaz, a qual combina amostragem, extração e concentração em uma única etapa. A SPME consiste na exposição de uma fibra, com um adsorvente ou uma mistura de adsorventes, em um headspace contendo os voláteis a serem investigados que serão, portanto, adsorvidos na fibra. Essa fibra se situa na ponta de uma agulha e antes e depois da amostragem ela é escondida dentro de um invólucro de aço para ser isolada do ar ambiente. ${ }^{10}$

Após a realização da etapa de adsorção, os analitos podem ser dessorvidos termicamente da fibra na porta de injeção de um cromatógrafo a gás, com espectrômetro de massas como detector (GC-MS), para a análise dos metabólitos voláteis produzidos (análise metabolômica).

Um dos parâmetros mais importantes a ser definido para uma extração eficiente por SPME é a escolha da melhor fibra. Pereira et al. ${ }^{11}$ avaliaram a eficácia de diferentes fibras na análise de voláteis de polpa de limão, kiwi, mamão e ameixa-pintada; nesse trabalho eles verificaram os revestimentos de poliacrilato (PA), polidimetilsiloxano (PDMS), polidimetilsiloxano/divinilbenzeno (PDMS/DVB), carbowax/divinilbenzeno (CW/DVB) e carboxen/polidimetilsiloxano (CAR/PDMS), sendo as de PDMS/DVB e CW/DVB as que forneceram melhores resultados. Outro trabalho interessante foi publicado por Pino, ${ }^{12}$ no qual foram testadas as fibras PDMS, PDMS/DVB, DVB/ CAR/PDMS, e CAR/PDMS para a extração de compostos voláteis em polpa de mamão. O autor observou que a fibra revestida com PDMS (não-polar) foi mais eficiente para a extração de ésteres do que a fibra revestida com PDMS/DVB (polar), enquanto essa última 
foi mais eficiente para a extração de álcoois do que aquela; por outro lado, a melhor eficiência geral de extração foi obtida quando a fibra revestida com DVB/CAR/PDMS foi utilizada.

Além da melhor fibra, outros parâmetros são de extrema importância para uma extração eficiente por SPME, como o tempo de acondicionamento para o acúmulo de voláteis no headspace e o tempo de exposição da fibra para a adsorção dos voláteis. Em 2017, Rocha et al. ${ }^{9}$ usaram um planejamento experimental para definir os níveis ótimos dos parâmetros tempo de acondicionamento (que os autores chamaram de tempo de equilíbrio) e tempo de exposição da fibra para extração de voláteis da polpa de mamão. Eles estudaram as faixas de 0 a 30 min para o tempo de equilíbrio $\left(\mathrm{T}_{\text {eq }}\right)$ e de 20 a 60 min para o tempo de exposição da fibra $\left(\mathrm{T}_{\text {exp }}\right)$; com os resultados, os autores definiram que o nível ótimo dos fatores $\mathrm{T}_{\mathrm{eq}}$ e $\mathrm{T}_{\text {exp }}$ seria de $15 \mathrm{~min}$ e $60 \mathrm{~min}$, respectivamente.

Apesar de ser possível encontrar alguns estudos na literatura que utilizaram SPME-GC-MS para análise de VOCs de mamão e outros alimentos, ${ }^{9,11-15}$ inclusive o publicado por Rocha et al. ${ }^{9}$ citado anteriormente, todos utilizavam a polpa do mamão, portanto, não se tratavam de análises não destrutivas (fruta inteira). $\mathrm{O}$ trabalho publicado por Terra et al. ${ }^{8}$ analisou amostras de fungos de mamão in vitro, ou seja, inoculados em meio BDA, e nenhuma otimização foi mostrada no trabalho. Após uma busca na literatura, não foram encontrados trabalhos de otimização de extração de voláteis de mamão de forma não destrutiva por SPME.

O presente trabalho propõe, portanto, estabelecer as melhores condições dos tempos de acondicionamento do mamão e de exposição da fibra para a extração de compostos voláteis por microextração em fase sólida de maneira não destrutiva, ou seja, utilizando o fruto inteiro sem nenhum dano na amostra. Para isso, foi utilizado um planejamento composto central (CCD- do inglês Central Composite Design). Esse estudo é importante, uma vez que análises de VOCs em frutas inteiras podem ser utilizadas em controle de qualidade para detecção precoce de microrganismos. Uma análise não destrutiva permite um menor número de etapas no processo analítico, sendo possível um aumento na velocidade da investigação e, ao mesmo tempo, uma diminuição nos erros operacionais, tornando o método mais confiável.

\section{PARTE EXPERIMENTAL}

\section{Amostras}

As amostras de mamões papaia utilizadas nesse trabalho foram adquiridas de supermercados de Campinas-SP, Brasil. Cuidados foram tomados ao selecionar amostras que não apresentavam injúrias físicas e sintomas de doenças pós-colheita.

Nos experimentos de otimização do processo de extração (determinação da melhor fibra e parâmetros de tempo de acondicionamento e de exposição da fibra) foram selecionados mamões em estágio de maturação em que menos de $15 \%$ da epiderme apresentava coloração amarela.

Por outro lado, no experimento de ajuste da programação de temperatura do forno durante a corrida cromatográfica foram utilizados mamões em estado de maturação mais avançado, com mais de $80 \%$ da epiderme com tonalidade amarela.

Todas as amostras foram pesadas e tiveram seus volumes verificados. As amostras mais semelhantes em termos de massa e volume foram selecionadas para a execução dos experimentos.

\section{Processo de extração de voláteis por SPME}

Mamões individuais foram inseridos em frascos de vidro de $2280 \pm 7 \mathrm{~mL}$, que foram hermeticamente fechados. Na tampa do frasco foi adaptado um septo de borracha para facilitar a inserção da agulha de SPME sem a perda de compostos voláteis. O sistema foi deixado em repouso para a liberação de voláteis no headspace por um tempo de acondicionamento. A amostragem dos voláteis foi feita, então, por um conjunto de SPME obtido da Supelco (Supelco park, Bellefonte, PA, EUA), com a exposição da fibra no headspace por um tempo determinado para a absorção dos VOCs. Durante todo processo de extração a temperatura foi controlada em $25 \pm 2{ }^{\circ} \mathrm{C}$. Após esse tempo, a agulha foi então removida do frasco e inserida na porta de injeção do GC-MS por 5 min para a dessorção dos compostos.

No início dos dias que seriam feitos os experimentos, foram realizadas limpezas das fibras a fim de remover quaisquer voláteis adsorvidos previamente nas mesmas, expondo-as na porta de injeção do GC-MS durante duas corridas completas.

\section{Condições cromatográficas iniciais}

Condições cromatográficas iniciais semelhantes às do trabalho de Zakaria et al., ${ }^{16}$ com algumas adaptações, foram empregadas, já que os autores utilizaram o mesmo cromatógrafo e a mesma coluna para análise de voláteis em manga. A temperatura do injetor foi matida a $250{ }^{\circ} \mathrm{C}$ e foi utilizado o modo splitless. O forno permaneceu a uma temperatura inicial de $40{ }^{\circ} \mathrm{C}$ durante $2 \mathrm{~min}$, aumentando gradativamente até $150{ }^{\circ} \mathrm{C}$ a uma taxa de $5{ }^{\circ} \mathrm{C} / \mathrm{min}$, mantendo-se nessa temperatura por $1 \mathrm{~min}$. Em seguida, elevou-se novamente a temperatura até $250{ }^{\circ} \mathrm{C}$ a uma taxa de $10{ }^{\circ} \mathrm{C} / \mathrm{min}$, que foi por fim mantida por $5 \mathrm{~min}$. O tempo total da corrida foi de $40 \mathrm{~min}$. A detecção no GC-MS foi feita usando $70 \mathrm{eV}$ de energia de ionização em uma faixa de massa de 50-550 a.m.u. A temperatura da fonte de íon foi de $280^{\circ} \mathrm{C}$ e da interface foi de $250{ }^{\circ} \mathrm{C}$. Hélio foi usado como gás de arraste a um fluxo de $1,2 \mathrm{~mL} / \mathrm{min}$.

\section{Seleção da melhor fibra}

Como se trata de um método novo de análise de voláteis em mamão, em que a extração não é feita da polpa, mas da fruta inteira, de maneira não destrutiva, faz-se necessário definir qual fibra é mais adequada para a adsorção de um maior número de compostos voláteis. A eficiência de dois tipos de fibra foi verificada: a revestida com PDMS $100 \mu \mathrm{m}$ e a com PDMS/DVB $65 \mu \mathrm{m}$, ambas adquiridas da Supelco (Supelco Park, Bellefonte, PA, USA). De acordo com o fabricante, as duas fibras são indicadas para análise de voláteis, sendo que a revestida de PDMS é mais indicada para análise de compostos menos polares de baixo peso molecular (MW 60-275), enquanto a fibra PDMS/DVB é indicada para extração de compostos mais polares como aminas, álcoois e compostos nitro-aromáticos (MW 50-300).

O procedimento de extração e análise dos VOCs foi feito em duplicada para cada fibra analisada, totalizando 4 experimentos. Os tempos de acondicionamento da fruta e de exposição da fibra foram de 5 min e $30 \mathrm{~min}$, respectivamente. Esses tempos foram definidos baseados em estudo prévio, no qual foi utilizado um planejamento CCD para definir as condições de extração de voláteis para a análise no GC-FID, sendo observado que apenas o tempo de exposição da fibra era significativo para o aumento do número de compostos detectados. ${ }^{17}$

\section{Mudanças nos parâmetros cromatográficos}

A fim de alcançar um menor tempo de corrida, sem diminuir o número de picos detectáveis pelo GC-MS, foram verificadas algumas combinações da programação da temperatura do forno do cromatógrafo com diferenças pequenas entre elas como mostrado na Tabela 1. 
Tabela 1. Programações de temperatura do forno do GC-MS

\begin{tabular}{|c|c|c|c|c|c|}
\hline ID & & $\begin{array}{c}\text { Taxa }\left({ }^{\circ} \mathrm{C} /\right. \\
\min )\end{array}$ & $\begin{array}{c}\text { Temperatura } \\
\left({ }^{\circ} \mathrm{C}\right)\end{array}$ & $\begin{array}{l}\text { Tempo de } \\
\text { espera (min) }\end{array}$ & $\begin{array}{c}\text { Tempo de } \\
\text { corrida (min) }\end{array}$ \\
\hline & Inicial & & 40 & 2 & 2 \\
\hline \multirow[t]{3}{*}{01} & Rampa 1 & 5 & 150 & 1 & 25 \\
\hline & Rampa 2 & 10 & 250 & 5 & 40 \\
\hline & Inicial & & 50 & 2 & 2 \\
\hline \multirow[t]{3}{*}{02} & Rampa 1 & 5 & 150 & 1 & 23 \\
\hline & Rampa 2 & 10 & 250 & 2 & 35 \\
\hline & Inicial & & 50 & 2 & 2 \\
\hline \multirow[t]{3}{*}{03} & Rampa 1 & 4 & 150 & 1 & 28 \\
\hline & Rampa 2 & 12 & 250 & 2 & 36,33 \\
\hline & Inicial & & 40 & 1 & 2 \\
\hline \multirow[t]{3}{*}{04} & Rampa 1 & 5 & 150 & 1 & 24 \\
\hline & Rampa 2 & 10 & 250 & 1 & 35 \\
\hline & Inicial & & 40 & 2 & 2 \\
\hline \multirow[t]{2}{*}{05} & Rampa 1 & 5 & 150 & 1 & 25 \\
\hline & Rampa 2 & 10 & 250 & 0 & 35 \\
\hline
\end{tabular}

Uma vez que um maior número de voláteis deveria ser detectado para observar também quando uma programação de temperatura ocasionaria uma sobreposição de picos, mamões em estado de maturação mais avançado foram utilizados. A melhor fibra foi empregada e os tempos de acondicionamento e de exposição da fibra também foram de $5 \mathrm{~min}$ e $30 \mathrm{~min}$, respectivamente.

\section{Planejamento Composto Central}

As condições de extração dos VOCs por SPME foram otimizadas usando um planejamento composto central (CCD), uma ferramenta estatística multivariada, já que métodos univariados seriam mais dispendiosos (um maior número de experimentos deveria ser feito) e não seria possível avaliar o efeito das interações entre os fatores. ${ }^{18-21}$ As variáveis selecionadas para a otimização do processo de extração por SPME foram o tempo de acondicionamento (tA, min) e o tempo de exposição da fibra (tExp, min), mantendo-se constante a temperatura (temperatura controlada $25 \pm 2{ }^{\circ} \mathrm{C}$ ).

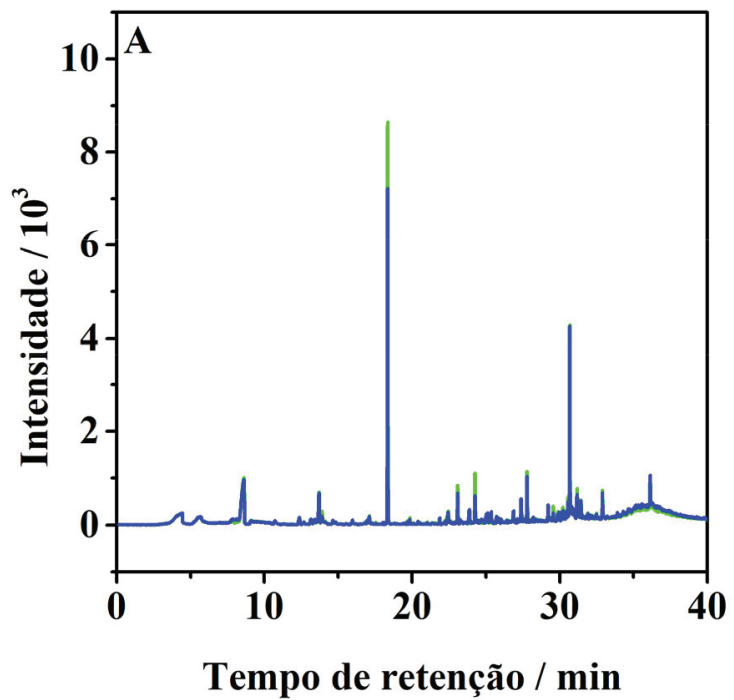

Os níveis de cada fator estudado estão mostrados na Tabela 2.

Tabela 2. Fatores e níveis estudados no CCD para a extração de voláteis por SPME

\begin{tabular}{lccccc}
\hline \multirow{2}{*}{ Variáveis } & \multicolumn{5}{c}{ Níveis } \\
\cline { 2 - 6 } & $-1,414$ & -1 & 0 & 1 & 1,414 \\
\hline tA / min & 9,65 & 20 & 45 & 70 & 80,35 \\
tExp / min & 13,79 & 20 & 35 & 50 & 56,21 \\
\hline
\end{tabular}

Treze experimentos foram gerados pelo planejamento CCD, sendo 4 pontos fatoriais, 4 axiais e 5 pontos centrais. Todos os experimentos foram realizados em ordem aleatória para minimizar os efeitos de fatores não controlados. A resposta para o planejamento foi o número de picos no cromatograma, e o objetivo foi a maximização da resposta. Os resultados obtidos foram submetidos à análise de variância (ANOVA) para avaliar a significância $(\mathrm{p}<0,05)$ da regressão, dos fatores individuais, da interação entre os fatores e a falta de ajuste. Em seguida, foi gerada uma superfície de resposta. A análise estatística foi feita utilizando o software Statistica (versão 13.0, StatSoft, Inc.) e planilhas eletrônicas do Microsoft ${ }^{\circledR}$ Excel. ${ }^{19}$ Com base nos resultados obtidos, foram selecionados os níveis ótimos de tA e tEF para as análises posteriores.

\section{RESULTADOS E DISCUSSÃO}

\section{Seleção da melhor fibra}

Apesar das condições analíticas idênticas, as duas fibras testadas (PDMS $100 \mu \mathrm{m}$ e PDMS/DVB $65 \mu \mathrm{m}$ ) têm espessuras e materiais de revestimento distintos e, consequentemente, polaridades diferentes. Dessa forma, diferentes analitos ou diferentes níveis de um mesmo analito são adsorvidos em cada uma das fibras. $\mathrm{O}$ resultado pode ser visualizado nos cromatogramas da Figura 1.

A coluna cromatográfica utilizada neste trabalho foi a HP-5MSUI. Trata-se de uma coluna apolar, ou seja, compostos menos polares ficam retidos por mais tempo na coluna e compostos mais polares são os primeiros a serem eluídos. Percebe-se, claramente, que entre os tempos de retenção 0 e 20 min mais compostos puderam ser detectados pelo GC-MS quando amostrados pela fibra revestida de PDMS/DVB $65 \mu \mathrm{m}$ (Figura 1 A). Os primeiros compostos eluídos

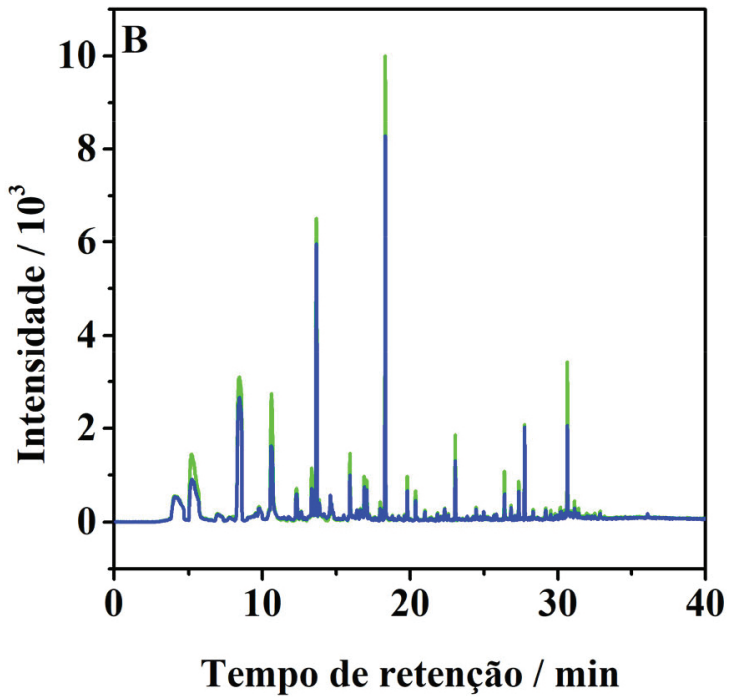

Figura 1. Cromatogramas obtidos para as amostragens do frasco com mamão na primeira (-) e na segunda repetição (-), utilizando as fibras PDMS (A) e $P D M S / D V B(B)$ 
- com menores tempos de retenção - são aqueles que possuem uma menor interação com a fase estacionária da coluna, ou seja, os compostos mais polares. A maioria desses compostos só pôde ser amostrada quando se utilizou a fibra PDMS/DVB. Por outro lado, após 30 min de corrida, um número maior de compostos foi detectado quando se utilizou a fibra PDMS que se caracteriza por ser uma fibra menos polar e, portanto, adsorvem mais eficientemente os analitos com a mesma característica. Todavia, a fibra PDMS/DVB foi selecionada por ser mais eficiente extraindo um maior número de compostos no total. Resultado semelhante foi obtido por Zakaria e colaboradores, ${ }^{16}$ que testaram quatro diferentes fibras e a revestida de PDMS/DVB $65 \mu \mathrm{m}$ foi a capaz de extrair o maior número de voláteis de manga. De acordo com os autores, isso pode ser explicado pelo fato de que a parte DVB da fibra PDMS/DVB consiste de poros largos (meso e macro) que podem contribuir para um rápido equilíbrio e levar a uma extração mais eficiente.

\section{Mudanças nos parâmetros cromatográficos}

O tempo de uma corrida completa com a programação de temperatura do forno até então utilizada (Ensaio 1) no GC-MS era de 40 min. Foram variados alguns parâmetros da programação de temperatura do forno, conforme descrito na Tabela 1, a fim de diminuir o tempo de corrida sem que o número de picos detectados fosse comprometido, com isso um número maior de análises poderiam ser realizadas em um menor período de tempo. O resultado está exposto na Figura 2.

Nota-se que o Ensaio 04 atingiu um tempo de corrida consideravelmente inferior ao do ensaio de referência (Ensaio 01) - 5 min a menos - e ainda foi detectado um número ligeiramente maior de picos -9 a mais. Nesse ensaio as temperaturas utilizadas e as rampas foram exatamente as mesmas do Ensaio 01, apenas os tempos de espera nas temperaturas inicial e final foram ambos diminuídos para 1 min. Essa condição do Ensaio 04 foi, então, escolhida para ser aplicada nos próximos experimentos do trabalho.

\section{Planejamento Composto Central}

Um planejamento composto central esférico foi utilizado para determinar as melhores condições de tempo de acondicionamento (tA) e tempo de exposição da fibra (tExp), utilizando-se a fibra

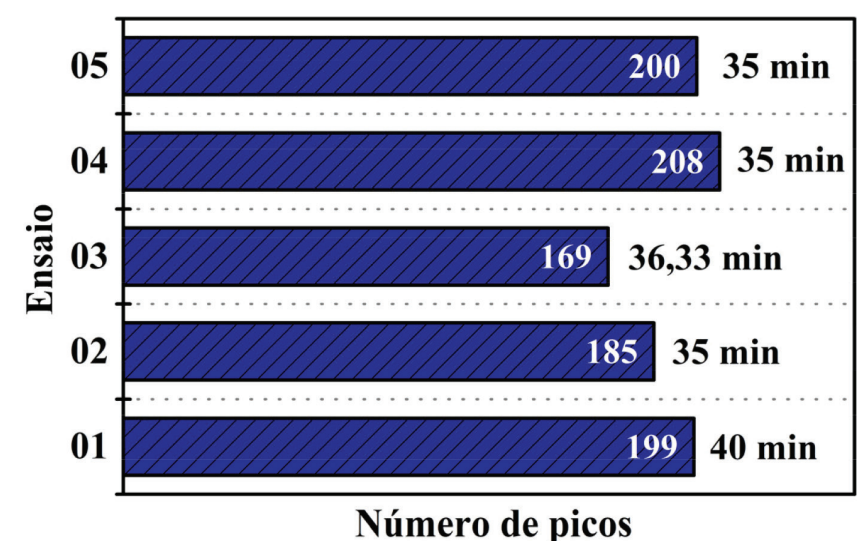

Figura 2. Número de picos detectados pelo GC-MS e tempo gasto na corrida em cada ensaio com diferentes programações de temperatura do forno

PDMS/DVB $65 \mu \mathrm{m}$. Neste trabalho, estudaram-se os níveis entre 9,6 e 80,36 min nos pontos axiais para o tA. Uma vez que a análise seria efetuada no fruto inteiro e possivelmente os voláteis se acumulariam de maneira mais lenta no headspace, o estudo da ampla faixa foi considerado viável. Para o tExp foi estudada a faixa de 13,79 a 56,21 min, referentes aos níveis $-\alpha$ e $+\alpha$, respectivamente. Esses níveis foram escolhidos com base em um estudo prévio em GC com detector por ionização de chama (FID - do inglês Flame Ionization Detector $)^{17}$ utilizando uma coluna mais polar: DB-23 da Agilent (Agilent Technologies, Palo Alto, CA, USA). Nesse estudo, percebeu-se que o número de picos detectados pelo GC-FID aumentava com o aumento do tempo de exposição da fibra entre 5,86 e 34,14 min, sendo que um máximo de resposta não foi atingido dentro da faixa estudada. Portanto, níveis maiores de tExp foram escolhidos para o presente trabalho. Entretanto, um limite que ainda era considerado razoável para uma boa velocidade de análise foi mantido. A Tabela 3 mostra os ensaios realizados e as respostas em números de picos para cada um deles.

O valor observado nos experimentos para o número total de picos variou entre 67 e 112, tais valores eram referentes aos experimentos de níveis $-\alpha$ e $+\alpha$, respectivamente do tExp, dando um indicativo de que essa variável é significativa para um aumento da resposta. Foi feita uma ANOVA e os resultados estão mostrados na Tabela 4.

Tabela 3. Planejamento experimental CCD codificado e decodificado utilizado para otimizar as condições de extração de compostos voláteis de mamão por SPME e as respostas em número de picos detectados de cada ensaio

\begin{tabular}{|c|c|c|c|c|c|c|}
\hline & \multirow{2}{*}{ ID } & \multicolumn{2}{|c|}{ Codificados } & \multicolumn{3}{|c|}{ Decodificados } \\
\hline & & $\mathrm{tA}$ & $\mathrm{tExp}$ & $\mathrm{tA} / \mathrm{min}$ & $\mathrm{tExp} / \mathrm{min}$ & Resposta (picos) \\
\hline \multirow{4}{*}{ Pontos fatoriais } & 01 & -1 & -1 & 20 & 20 & 69 \\
\hline & 02 & 1 & -1 & 70 & 20 & 83 \\
\hline & 03 & -1 & 1 & 20 & 50 & 102 \\
\hline & 04 & 1 & 1 & 70 & 50 & 95 \\
\hline \multirow{4}{*}{ Pontos axiais } & 05 & $-\alpha$ & 0 & 9,64 & 35 & 86 \\
\hline & 06 & $+\alpha$ & 0 & 80,36 & 35 & 105 \\
\hline & 07 & 0 & $-\alpha$ & 45 & 13,79 & 67 \\
\hline & 08 & 0 & $+\alpha$ & 45 & 56,21 & 112 \\
\hline \multirow{5}{*}{ Pontos centrais } & 09 & 0 & 0 & 45 & 35 & 91 \\
\hline & 10 & 0 & 0 & 45 & 35 & 91 \\
\hline & 11 & 0 & 0 & 45 & 35 & 97 \\
\hline & 12 & 0 & 0 & 45 & 35 & 98 \\
\hline & 13 & 0 & 0 & 45 & 35 & 91 \\
\hline
\end{tabular}


A

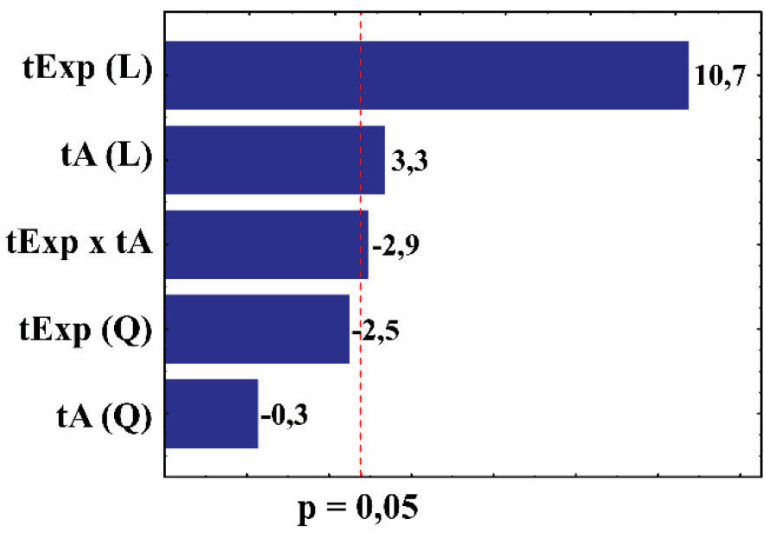

B

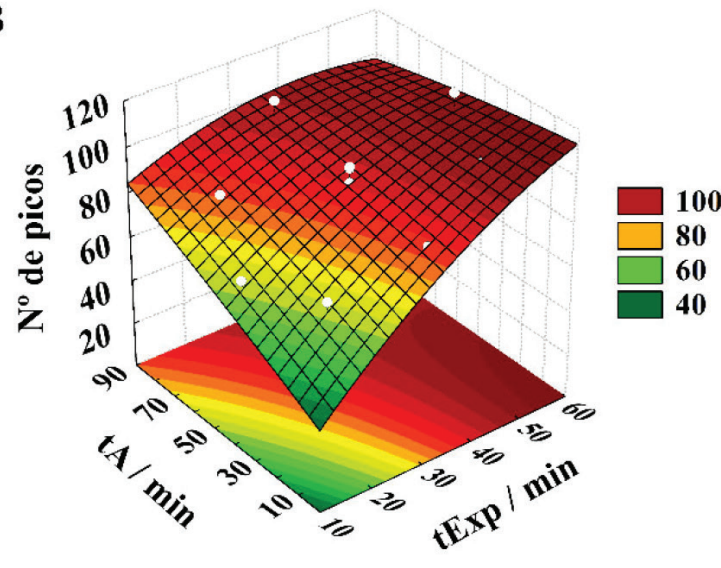

Figura 3. Gráfico de Pareto (A) e superfície de respostas (B) referente ao planejamento CCD para a otimização dos níveis dos fatores tExp e tA

Tabela 4. Análise de variância dos resultados obtidos do CCD para otimizar as condições de extração de compostos voláteis de mamão por SPME

\begin{tabular}{lccccc}
\hline & SS & df & MS & F & p \\
\hline tExp (L) & 1475,3 & 1 & 1475,3 & 115,26 & 0,0004 \\
tExp (Q) & 78,7 & 1 & 78,7 & 6,15 & 0,0683 \\
tA (L) & 143,4 & 1 & 143,4 & 11,20 & 0,0286 \\
tA (Q) & 0,9 & 1 & 0,9 & 0,071 & 0,8026 \\
tExp x tA & 110,2 & 1 & 110,2 & 8,61 & 0,0426 \\
Falta de ajuste & 147,9 & 3 & 49,3 & 3,85 & 0,1128 \\
Erro puro & 51,2 & 4 & 12,8 & & \\
Total SS & 2006,8 & 12 & & & \\
\hline
\end{tabular}

A tabela dos resultados da ANOVA confirma que o termo linear do tExp é bastante significativo para a mudança da resposta com valor p muito menor que 0,05 (intervalo de confiança de $95 \%$ ). Além disso, o termo linear do tA e a interação entre as variáveis tExp e tA também foram significativas. Todavia, os termos quadráticos de ambas variáveis não foram significativos para uma mudança na resposta. A Figura 3 ilustra melhor os resultados do CCD com o gráfico de pareto dos efeitos padronizados e a superfície de resposta contruída.

Pelo gráfico de Pareto (Figura 3 A) a significância de cada fator pode ser melhor visualizada. Percebe-se que enquanto os termos lineares do tExp e tA foram positivamente significativos - um aumento nos níveis dessas variáveis ocasiona um aumento na resposta - a interação entre eles foi negativamente significativa, ou seja, um aumento do nível das duas variáveis concomitantemente acarreta em uma diminuição da resposta. Isso pode ter ocorrido devido a uma saturação da fibra quando o tA e o tExp são muito altos. Pela superfície de resposta obtida (Figura 3 B), percebe-se que um nível máximo não foi atingido e que talvez o número de picos detectáveis continuasse aumentando se fosse aumentado o tExp, mantendo o tempo de acondicionamento baixo, por volta de $10 \mathrm{~min}$, por exemplo. Entretanto, após $45 \mathrm{~min}$ de tExp a mudança na resposta não é tão grande, sendo que nesse ponto $(\mathrm{tA}=10$ min e $\mathrm{tExp}=45 \mathrm{~min})$ já são amostrados por volta de 100 compostos detectáveis por GC-MS. Dessa forma, 10 min de tA e 45 min de tExp foram selecionados como as melhores condições para a extração não destrutiva de voláteis de mamão.

\section{CONCLUSÕES}

A metodologia desenvolvida utilizando microextração em fase sólida mostrou ser eficiente para a extração de voláteis de mamão de maneira não destrutiva e não invasiva. A fibra PDMS/DVB de SPME apresentou uma maior eficiência na extração dos metabólitos voláteis de mamão papaia. A otimização por planejamento composto central das condições de extração permitiu a definição melhor do tempo de acondicionamento e do tempo de exposição da fibra. Ambas variáveis estudadas e a interação entre elas foram significativas para a mudança na resposta (número de picos detectados). A melhor condição encontrada após a otimização permitiu a detecção de mais de 100 compostos por GC-MS.

\section{AGRADECIMENTOS}

Agradecemos o apoio financeiro da Fundação de Amparo à Pesquisa do Estado de São Paulo - Brasil (FAPESP - Processos número 2019/10043-5 e 2018/25318-7) e à Coordenação de Aperfeiçoamento de Pessoal de Nível Superior - Brasil (CAPES) código financeiro 001. Agradecemos também à Debora Renata Cassoli de Souza Dutra pelo apoio experimental no instrumento GC-MS.

\section{REFERÊNCIAS}

1. Lima, R. C. A.; Lima, J. A. A.; Souza Jr., M. T.; Pio-Ribeiro, G.; Andrade, G. P.; Fitopatol. Bras. 2001, 26, 689.

2. Annegowda, H. V; Bhat, R.; Yeong, K. J.; Liong, M.; Karim, A. A.; Bhat, R.; Yeong, K. J.; Liong, M.; Karim, A. A.; Int. J. Food Prop. 2014, 17, 283.

3. http://www.fao.org/faostat/en/\#data/QC/visualize, acessada em julho 2020.

4. http://www.fao.org/faostat/en/\#data/TP/visualize, acessada em julho 2020.

5. Gustavsson, J.; Cederberg, C.; Sonesson, U.; Emanuelsson, A. Em The methodology of the FAO study: "Global Food Losses and Food Waste extent, causes and prevention" - FAO, 2011; Gustavsson, J., Cederberg, C., Sonesson, U., eds.; SIK, 2013.

6. Oliveira, J. G. de; Vitória, A. P.; Food Res. Int. 2011, 44, 1306.

7. Encinas-basurto, D.; Valenzuela-quintanar, M. I.; Sánchez-estrada, A.; Tiznado-hernández, M. E.; Rodríguez-Félix, A.; Troncoso-Rojas, R.; Chil. J. Agric. Res. 2017, 77, 194.

8. Terra, L. R.; Queiroz, S. C. N.; Terao, D.; Ferreira, M. M. C.; J. Chemom. (2020) https://doi.org/10.1002/cem.3244

9. Rocha, R. F. J.; da Silva Araújo, Í. M.; de Freitas, S. M.; dos Santos Garruti, D.; J. Food Sci. Technol. 2017, 54, 4042.

10. Xu, C.; Chen, G.; Xiong, Z.; Fan, Y.; Wang, X.; Trends Anal. Chem. 2016, 80, 12.

11. Pereira, J.; Pereira, J.; Câmara, J. S.; Talanta 2011, 83, 899. 
12. Pino, J. A.; Food Chem. 2014, 146, 120.

13. Balbontín, C.; Gaete-Eastman, C.; Vergara, M.; Herrera, R.; Moya-León, M. A.; Postharvest Biol. Technol. 2007, 43, 67.

14. Lieb, V. M.; Esquivel, P.; Cubero Castillo, E.; Carle, R.; Steingass, C. B.; Food Chem. 2018, 248, 238.

15. Ribeiro, J. S.; Teófilo, R. F.; Augusto, F.; Ferreira, M. M. C.; Chemom Intell. Lab. Syst. 2010, 102, 45.

16. Zakaria, S. R.; Tajuddin, R.; Osman, R.; Saim, N.; Saaid, M.; Sci. Technol. 2017, 25, 167.
17. Terra, L. R.; Queiroz, S. C. N.; Terao, D.; Ferreira, M. M. C.; Resumos do X Workshop de Quimiometria, Salvador, Brasil, 2019.

18. Bezerra, M. A.; Santelli, R. E.; Oliveira, E. P.; Villar, L. S.; Escaleira, L. A.; Talanta 2008, 76, 965.

19. Teófilo, R. F.; Ferreira, M. M. C.; Quim. Nova 2006, 29, 338.

20. Breitkreitz, M. C.; Souza, A. M. de; Poppi, R. J.; Quim. Nova 2014, 37, 564.

21. Pereira, F. M. V.; Pereira-Filho, E. R.; Quim. Nova 2018, 41, 1061. 